\title{
Integrating elements in an $i$-DSS for portfolio management in the Mexican market
}

\author{
M. A. Osorio ${ }^{1}$, A. Sánchez ${ }^{2}$ \& M. A. Gómez ${ }^{3}$ \\ ${ }^{1,2}$ School of Computer Sciences, Universidad Autónoma de Puebla, \\ Ciudad Universitaria, Puebla, México \\ ${ }^{3}$ Actuarial Sciences and Statistics Department, \\ Universidad de las Américas Puebla, Cholula, México
}

\begin{abstract}
We present the main elements to design an intelligent decision support system ( $i$-DSS) for portfolio management in the Mexican market, including the financial investment considerations for a knowledge database and the requirements for a portfolio optimization model. We take into account the tax impact and the Mexican market conditions and volatility into the models; and modify the classic Markowitz model, augmenting constraints to propose stochastic linear and stochastic quadratic models. The extended Markowitz model is solved with an efficient adapted Evolutionary Simulated Annealing algorithm. The efficient frontier is calculated using the augmented model. We present a proposal for the integration of an $i$-DSS (intelligent Decision Support Systems) with the knowledge and preference system, the optimization model and the Mexican market characteristics.

Keywords: intelligent decision support systems, portfolio optimization.
\end{abstract}

\section{Introduction}

Intelligent Decision Support Systems ( $i$-DSS), as successors of Management Information Systems (MIS), traditionally follow the decision logic line of thinking and include in MIS algorithmic tools to improve the choice activity of decision makers. This includes optimization methods, mathematical programming, multi criteria models etc. They are "structure related", normally assume that the decision problem can be formulated mathematically and do not stress information processing and display. "Knowledge" is represented in many 
different ways, such as, frames, semantic nets, rules etc. Knowledge is processed in inference machines, which normally perform symbol processing, i.e., truth values of antecedents, conclusions etc. Some experts consider ES as part of $i$-DSS [1]; others see $i$-DSS and ES as basically different systems and others combine the two approaches into "Knowledge-based DSS" [2].

In this paper, we approach the design of decision support systems for portfolio management in Mexican market. In section 2, we describe the capabilities and potentialities of intelligent decision support systems. The Mexican market is described in section 3, including definitions, history, etc. In section 4 we describe the elements for a portfolio optimization model, taking into account the Mexican market conditions and volatility into the constraints in the model. Section 5 describes in detail an evolutionary simulated annealing algorithm that proved to be efficient in solving the Markowitz's models. The conclusions in section 6, present an integration of the proposed stochastic linear and stochastic quadratic models in an $i$-DSS framework.

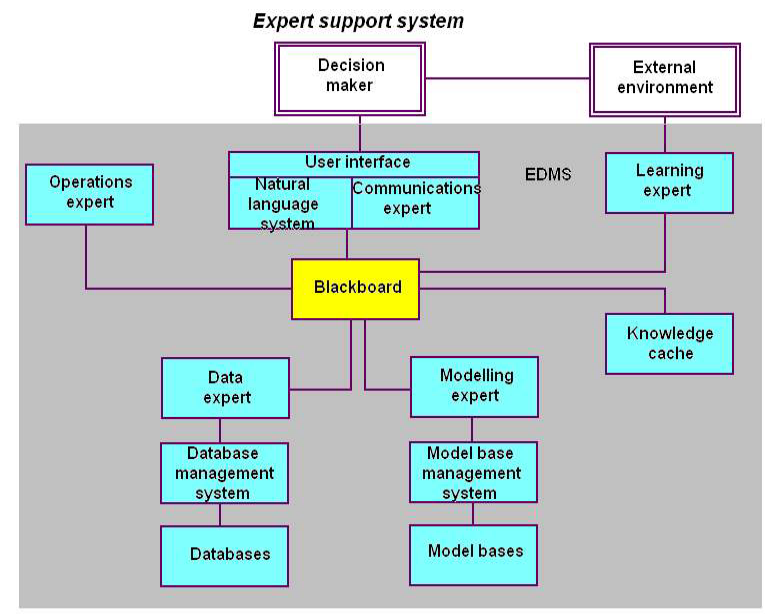

Figure 1: $\quad$ Expert support system [3].

\section{Intelligent decision support systems}

The idea of making "intelligent" DSS ( $i$-DSS) is supported by most major researchers in the field. The resulting system has been referred by different authors. It has been called an $i$-DSS, and intelligent support system, and expert $i$-DSS (EDSS), and a know-ledge-based $i$-DSS ( $i$-KBDSS). It has been suggested that an $i$-DSS could support higher levels of decision making if each of its three subsystems is enhanced by AI. For example, knowledge representation techniques could be used to enhance the data subsystem; a natural language interface could serve as a front-end to the dialogue subsystem; and reasoning rules could strengthen the model subsystem. The main forms of integration are: 
- ES integration intro various $i$-DSS components.

- ES integration as a separate component of the $i$-DSS.

- $i$-DSS models and a data access as a component of an ES.

In some cases, there are other problems with $i$-DSS-ES integration; integrating a general problem support system with a specific narrow problem can be a formidable task. It is not clear that the expertise in ES is always transferable across problem domains as an $i$-DSS should support. Furthermore, the time and cost involved in the design and development of such an interesting system may be difficult to justify. A new form of integration is necessary to deal with the preceding problems. The new model of $i$-DSS-Es integration is called an expert support system (ESS) [3] (see Fig. 1).

\section{Mexican market}

In the early 1990's the Mexican authorities (officially) lifted capital controls on foreign exchange transactions. Other financial market reforms came about at the same time. However, institutional participants remained subject to tight investment regimes precluding them from adding foreign assets to their portfolios. Since 2003 mutual funds can invest foreign stocks, bonds and other mutual funds. Since mid 2003, the Mexican's Securities Market: BMV (Bolsa Mexicana de Valores) established a UTP platform (Unlisted Trading Privileges for foreign stocks, equity index ETFs (Exchange Traded Funds) and fixed income ETFs. Since early 2004, pension funds can operate equity, foreign exchange and fixed income derivatives. In March 2004, Mexico started with an options market and in November 2004, pension funds started investing in domestic equities and foreign stocks and bonds. Savings through SIEFORES reaches $55 \%$ of traditional savings.

Table 1: Capital controls and financial regulation.

\begin{tabular}{l|llll}
\hline Period & Controls on Public & Bank & Institutional Investors & Mutual Funds \\
\hline $1976-1982$ & No & No & No & N.A. \\
$1982-1991$ & Yes & Yes & Yes & Yes \\
$1991-2002$ & No & Yes & Yes & Yes \\
$2002-2003$ & No & Yes & Yes & No \\
\hline $\mathbf{2 0 0 4}$ & No & Yes & No & No \\
\hline
\end{tabular}

Even if the impact on Mexico's economic growth has not been properly measured, according to other experiences, these changes can contribute to the development of Mexico's capital market, even if some people think that this liberalization may make Mexico more vulnerable to changes in domestic and external macroeconomic conditions. Based on other experiences, adding equities and foreign instruments to the set of eligible assets will induce better rates on Mexican Treasures and corporate bond yields will tend to go up (Reynoso [7]).

The trend to lift capital controls has the purpose to develop the local intermediation industry, according to experiences that say that cross listings in domestic and foreign markets adds to liquidity and market development, lifting 
controls on local investors can positively influence national savings, productivity and growth and may be good for the economy. The global market for the BMV has already 200 stocks and ETFs and includes: 31 stocks of the DJI index, 48 stocks of the DJ STOXX50 Index, 20 NASDAQ listed stocks, 78 NYSE and AMEX listed stocks, 5 TSX listed stocks, 9 Exchange Traded Funds.

The global market components for the BMV for onshore and offshore (cross border) stocks can be seen in Figs. 2 and 3.

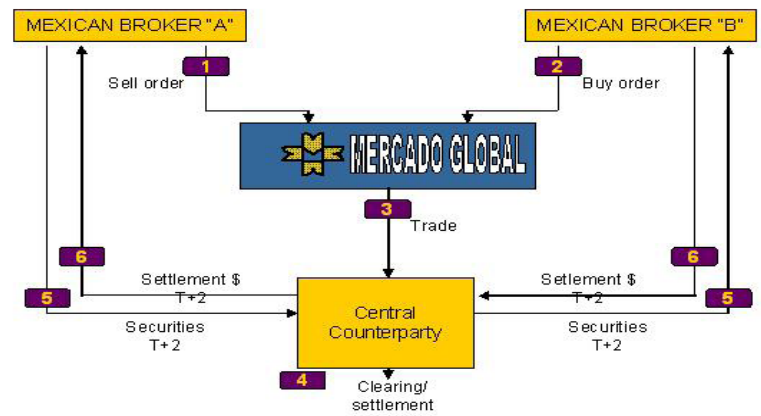

Figure 2: Global market BMV: onshore stocks.

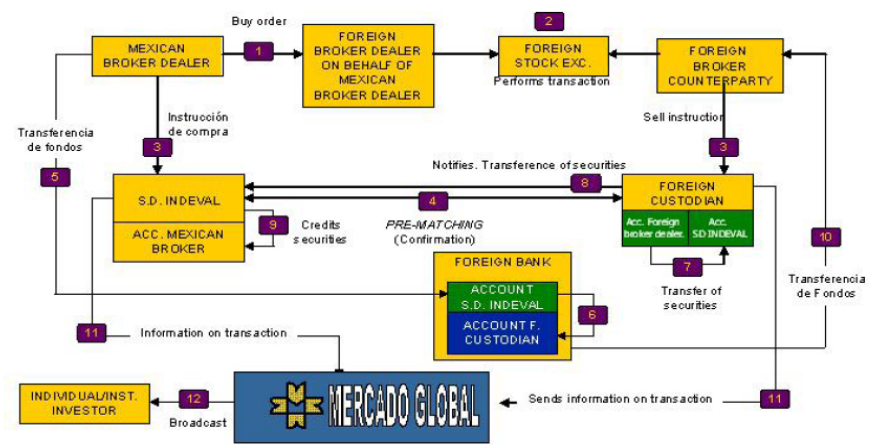

Figure 3: Global market BMV: offshore stocks.

Has it been a good strategy to invest in the BMV? To answer this question we will explore BMV's history [7]. Fig. 4 shows the investment's index evolution taking in consideration the period: 1980-2004. Trends show that the index's growth has been very high in the long term even if the growth is lower in the short term.

The BMV's risk return frontier shows in Fig. 5 that diversification plays an important role in the expected returns. As more stocks are included in the investment, as higher is the expected return for the same risk level.

The BMV individual stocks' growth for the period 1996-2004 is shown in Table 2. It can be seen that most of the stocks have had a stable behavior and a positive growth. 


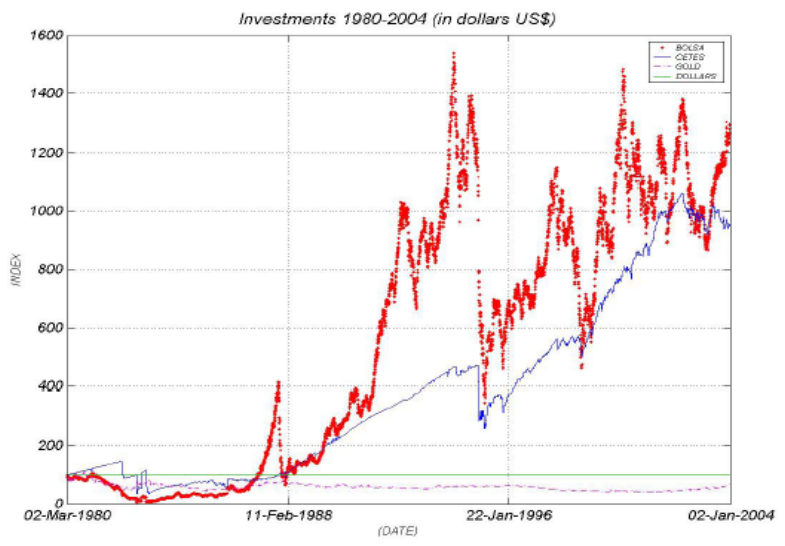

Figure 4: Index evolution: 1980-2004.

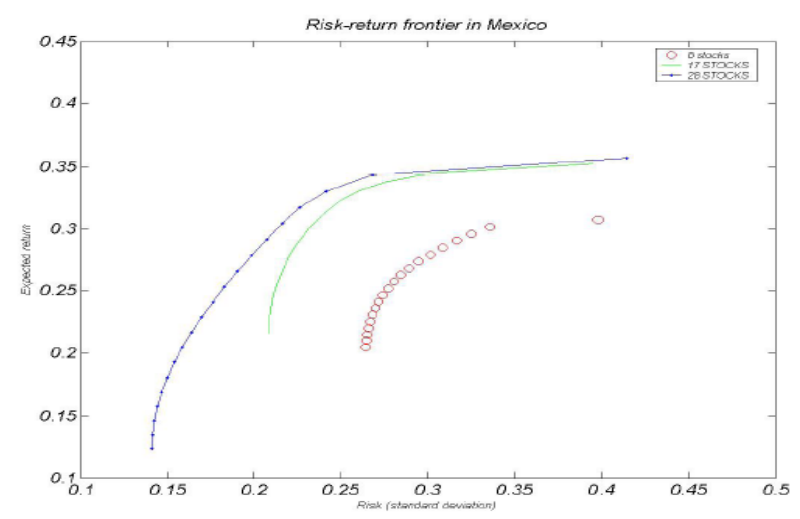

Figure 5: $\quad$ BMV's risk-return frontier.

Conditions for the BMV are changing. The new regulation will likely achieve the objective of significantly improving the risk-return mix available to domestic investors. Allowing institutional investors to include domestic and foreign equities in their portfolios will translate into a much larger demand for foreign securities than local stocks. The regulation will effectively impede the amplification of exogenous shocks via the impact of the portfolio composition of pension funds on the balance of payments.

\section{Financial investment elements}

The definitions and elements that should be present in the knowledge database, considering taxes and the investor's scenario can be reviewed to detail in [4]. They were first introduced in the model by Osorio and Sanchez [5] and Osorio et al. [6]. 
Table 2: $\quad$ Individual stocks in the BMV (1996-2004).

\begin{tabular}{|l|l|l|l|l|l|}
\hline Portfolio num. & 1 & 3 & 5 & 7 & 9 \\
\hline BIMBO & 0.0357 & 0.0322 & 0.0287 & 0.0251 & 0.0244 \\
\hline CEMEX & -0.0000 & -0.0000 & -0.0000 & -0.0000 & -0.0000 \\
\hline COMERCI & -0.0000 & -0.0000 & -0.0000 & 0.0000 & 0.0000 \\
\hline CONTAL & 0.2119 & 0.2118 & 0.2117 & 0.2116 & 0.2115 \\
\hline DESC & 0.0000 & 0.0000 & 0.0000 & 0.0000 & 0.0000 \\
\hline BANCOMER & 0.0215 & 0.0252 & 0.0289 & 0.0327 & 0.0335 \\
\hline BANORTE & 0.0907 & 0.0935 & 0.0962 & 0.0990 & 0.0996 \\
\hline GISSA & -0.0000 & -0.0000 & -0.0000 & -0.0000 & -0.0000 \\
\hline ICA & -0.0000 & -0.0000 & -0.0000 & -0.0000 & -0.0000 \\
\hline KIMBER & 0.0000 & 0.0000 & 0.0000 & 0.0000 & 0.0000 \\
\hline KOF & 0.2200 & 0.2222 & 0.2244 & 0.2268 & 0.2272 \\
\hline MASECA & -0.0000 & -0.0000 & -0.0000 & -0.0000 & -0.0000 \\
\hline PENOLES & 0.0151 & 0.0136 & 0.0121 & 0.0106 & 0.0103 \\
\hline SAVIA & -0.0000 & -0.0000 & -0.0000 & -0.0000 & -0.0000 \\
\hline SORIANA & 0.0068 & 0.0062 & 0.0055 & 0.0048 & 0.0047 \\
\hline TELMEX & 0.2550 & 0.2558 & 0.2567 & 0.2575 & 0.2577 \\
\hline TELEVISA & -0.0000 & -0.0000 & -0.0000 & -0.0000 & -0.0000 \\
\hline GFINBUR & 0.0000 & 0.0000 & -0.0000 & -0.0000 & -0.0000 \\
\hline GMODELO & 0.0471 & 0.0425 & 0.0379 & 0.0331 & 0.0322 \\
\hline ELEKTRA & -0.0000 & -0.0000 & -0.0000 & -0.0000 & -0.0000 \\
\hline GMEXICO & 0.0000 & 0.0000 & 0.0000 & 0.0000 & 0.0000 \\
\hline CIE & 0.0848 & 0.0868 & 0.0887 & 0.0908 & 0.0912 \\
\hline TELECOM & 0.0114 & 0.0103 & 0.0092 & 0.0080 & 0.0078 \\
\hline
\end{tabular}

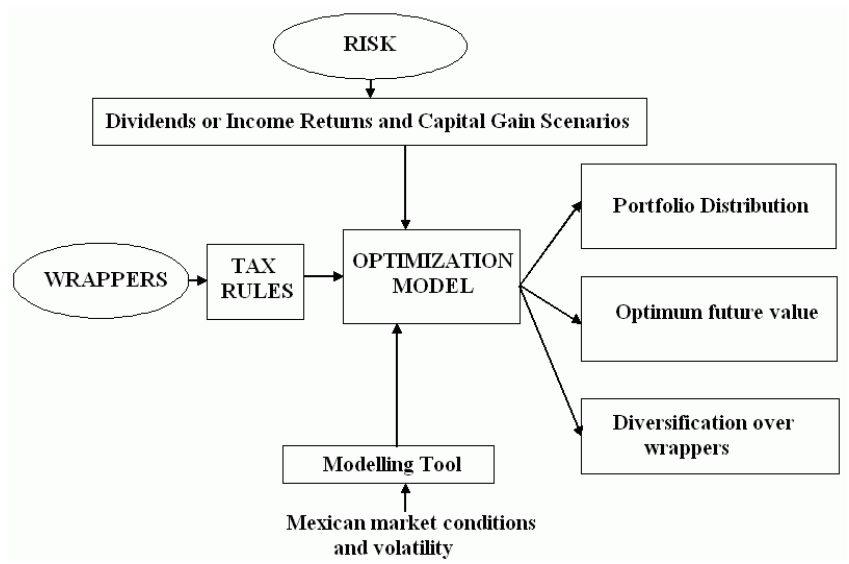

Figure 6: Elements for a portfolio optimization model.

From a global point of view, we should consider the elements shown in Fig. 6 in a portfolio optimization model. The elements for a portfolio optimization model are constraints and uncertainty representation in scenario trees. The constraints in the model are:

- Initial Allocation

- Cash Balance Equations

- Wealth Balance 
- Cumulative Taxes

- Cumulative Returns

- Diversification Constraints

These models require a coherent representation of uncertainty. This is expressed in terms of multivariate continuous distributions. Hence, a decision model is generated with internal sampling or a discrete approximation of the underlying continuous distribution. The random variables are the uncertain return values of each asset on an investment. The discretization of the random values and the probability space leads to a framework in which a random variable takes finitely many values. At each time period, new scenarios branch from the old, creating a scenario tree. Scenario trees can be generated based on different probabilistic approaches as simulation or optimization as presented in Osorio et al. [6].

\section{An evolutive simulated annealing algorithm for the extended Markowitz model}

The problem of optimally selecting a portfolio among $\mathrm{n}$ assets was formulated by Markowitz in 1952 as a constrained quadratic minimization problem [10]. The main assumption on Markowitz's model is that the aim of the investor is to design a portfolio which minimizes risk while achieving a predetermined expected return. An investor should be compensated with an increase in the portfolio's return if she accepts an increased level of risk.

The model considers that each asset is characterized by a return varying randomly with time. The variance of its return measures the risk of each asset. If each component $\mathrm{x}_{\mathrm{i}}$ of the vector $\mathrm{X}=\left\{\mathrm{x}_{1}, \mathrm{x}_{2}, . ., \mathrm{x}_{\mathrm{n}}\right\}$ represents the proportion of an investor's wealth allocated to asset $i$ and $r_{i}$ is the individual asset return contained in a vector $\mathrm{R}=\left\{\mathrm{r}_{1}, \mathrm{r}_{2}, . ., \mathrm{r}_{\mathrm{n}}\right\}$, then the total return of the portfolio is given by the scalar product of $\mathrm{X}$ by R. The set of optimal solutions of the Markowitz's model, parameterized over all possible values of the expected return constitutes the mean-variance frontier of the portfolio. The basic mean-variance model has been studied extensively but is often too simplistic to represent the complexity of real-world portfolio selection problems in an adequate fashion. In order to enrich the model, we introduced more realistic constraints mentioned above (see [4]). With these additional constraints the resulting problem is a mixed integer and combinatorial problem so the decision of which assets to include or not is a crucial one which turns out to be NP hard problem.

The use of a hybrid search algorithm that combines a simulated annealing (SA) procedure and some elements taken from evolutionary strategies to find a set of solutions for the mean-variance frontier, proved to be very efficient to approach this problem [11].

Unlike the standard version of SA where just one portfolio is considered this algorithm uses a whole "population" of portfolios which are permanently ranked. According to evolutionary principles, the best of the population's members are favored whereas the worst are eliminated and replaced either by a clone of one of 
the best portfolios or some new individual which is endowed with properties of the best portfolios.

The algorithm starts with a random initialization of a solution each representing a portfolio. This includes selecting $\mathrm{k}$ of the $\mathrm{N}$ available assets and assigning them random positive weights $\mathrm{w}_{\mathrm{i}}$ such that their sum adds up to 1 ; i.e., $\mathrm{w}_{\mathrm{i}}$ is the weight of asset $\mathrm{i}$ in a portfolio during iteration $t$.The subsequent iterations consist of three stages: evaluation and ranking of the portfolios; modification of the portfolio structure; replacement of the poorest portfolios in the population.

Evaluation ranks the solutions according to their fitness. This evaluation and ranking procedure is important to decide which solutions to reinforce and which to eliminate. Modification process uses the set of assets contained in a portfolio and changes the weights originally assigned to each one according to investor's rules and, with random probabilities, it may incorporate new elements to the portfolio. Having changed the weights and standardized them such that the sum of $\mathrm{w}_{\mathrm{i}}$ is equal to one, the fitness of the modified portfolio $\mathrm{P}$ is calculated according to the principles of SA, the modifications are accepted with probability $\mathrm{p}$ which comes from the Metropolis function. Replacement is used to reinforce promising tendencies on the one hand and eliminate rather disappointing ones on the other. The worst portfolios of the current population are replaced with new portfolios which are considered to have high potential. We distinguish two alternatives of high potential replacements: Clone and Average idol. In clone, the probabilities for selecting an existing portfolio are calculated such that the better a prodigy's fitness the higher the probability it is chosen. In average idol, an average weight for each asset is calculated based on the elitists and prodigies portfolios.

The algorithm finishes when a fixed number of iterations is reached and the best solution, which corresponds to the elitist portfolio is reported. The process is repeated for each required value of the return in order to create the meanvariance frontier.

\section{6 i-DSS proposed}

The elements in the portfolio optimization models and the knowledge databases can be integrated in an $i$-DSS scheme, showed in Fig. 7. The scheme presented is based in the scheme first introduced by Trippy and Lee [9], but instead of prioritized variables we generated additional constraints with enriched elements to manage a wide range of withdrawals in different wrappers for the portfolio elements considered, took into account the investors' scenario first introduced by Osorio et al. [5], and introduce the Mexican market conditions and volatility into the knowledge and preference system.

\section{Examples and conclusions}

We considered an example where an investor sold a factory, would like to invest $\$ 8,000,000$ and to live from this money the next eleven years. He would like to 
get an annual withdrawal of $\$ 500,000$. How would he have to invest his money in order to maximize the money he will receive back after eleven years?

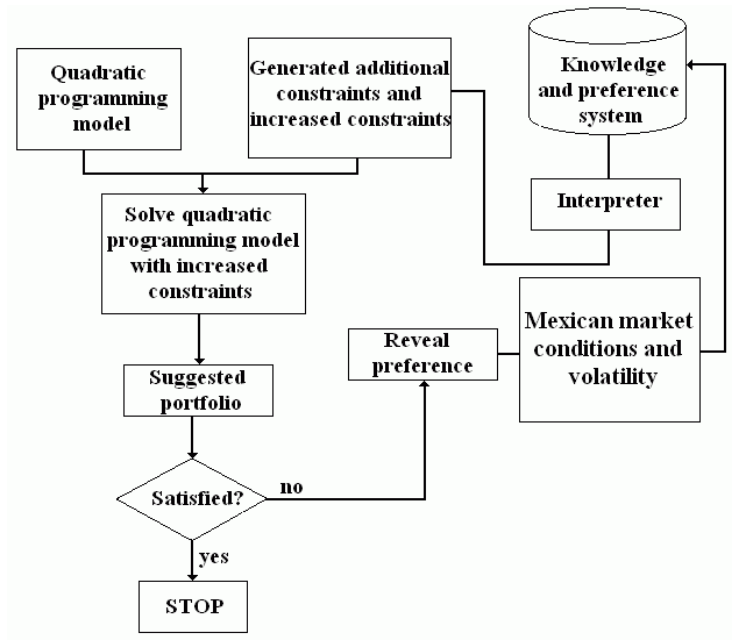

Figure 7: $\quad i$-DSS that integrates knowledge and portfolio optimization models.

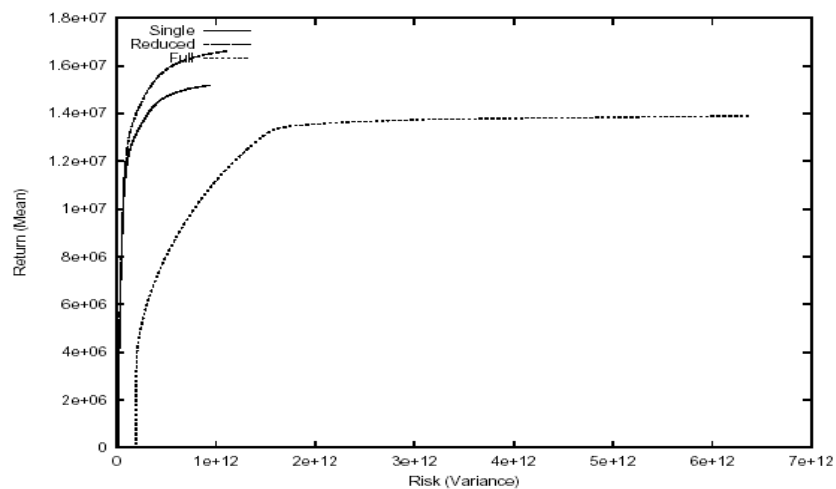

Figure 8: Impact of scenario trees.

Results show that scenario tree structure plays an important role in the efficient frontier obtained by the Markowitz models, as can be seen in Fig. 8. In the example, we compared a one branch scenario tree with a tree with four branches for the first period and one branch for the rest, and with a full binary tree.

The impact of real life situations in the efficient frontier can be seen in Fig. 9. We introduced the options of gift, inheritance and emigration. Finally, we can conclude that the integration of knowledge in portfolio optimization models can help to develop flexible and efficient $i$-DSSs for investment management. 


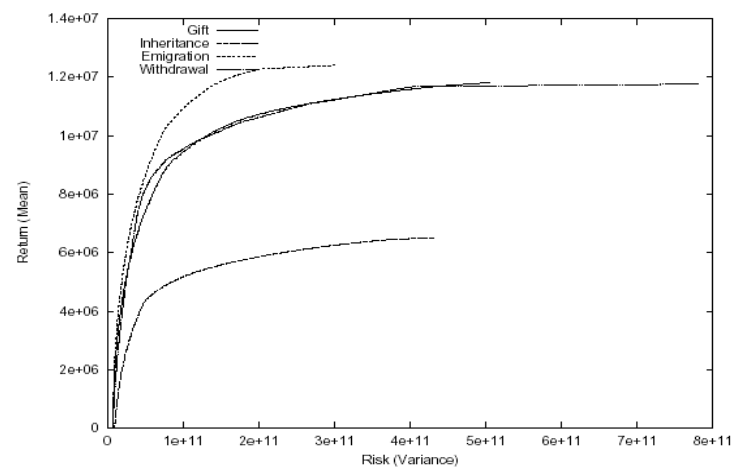

Figure 9: Impact of real life situations.

\section{References}

[1] Turban, E.: "Decision Support and Expert Systems", $2^{\text {nd }}$ Ed., Macmillan, New York (1988)

[2] Klein, R. L., Methlie, L. B.: "Knowledge-Based Decision Support Systems", $2^{\text {nd }}$ Ed., Wiley (1995)

[3] El-Najdawi, M. K., Stylianou, A. C.: "Expert Support Systems: Integrating AI Technologies", Communications of the ACM, Vol. 36, No. 12, (1993) 55-103

[4] Osorio L. M., Sánchez L. A.: “Designing Decision Support Systems for Portfolio Management", Technical Report, School of Computer Sciences, Universidad Autónoma de Puebla (2005)

[5] Osorio M. A., Settergren R., Rustem B., Gulpinar N.: "Post Tax Optimal Investments, Financial Engineering, e-Commerce and Supply Chain", Kluwer Academic Publishers, (2002) 153-173

[6] Osorio M. A., Gulpinar N., Settergren R., Rustem B.: "Post-Tax Optimization with Stochastic Programming", European Journal of Operational Research, Vol. 157, (2004) 152-168

[7] Reynoso A.: “Opening up a Securities Market: Mexico's New Push for Liberalization 2003-2004", Mexican Stock Exchange, SCID Latin America Conference (2004)

[8] Reynoso A.: "Can Subsidiaries of Foreign Banks Contribute to the Stability of the Forex Market in Emerging Economies? A Look at some Evidences from the Mexican Financial System", Working paper 8864, National Bureau of Economic Research, Cambridge, MA (2002)

[9] Trippy, R., Lee, J.: “Artificial Intelligence in Finance and Investing”, IRWIN Professional Publishing (1996)

[10] Markowitz, H. M.: "Portfolio selection”, Cambridge, MA, Blackwell. (1959)

[11] Mora, X., Gomez, M. A.: “An evolutive simulated annealing algorithm for the enriched Markowitz's Model", Technical Report, Actuarial and Statistics department, Universidad de las Américas Puebla (2004). 